\title{
Effect modification of the association between total cigarette smoking and ALS risk by intensity, duration and time-since-quitting: Euro-MOTOR
}

\author{
Susan Peters, ${ }^{\oplus 1,2}$ Anne E Visser, ${ }^{2}$ Fabrizio D’Ovidio, ${ }^{3}$ Jelle Vlaanderen, ${ }^{1}$ \\ Lützen Portengen, ${ }^{1}$ Ettore Beghi, ${ }^{\oplus 4}$ Adriano Chio, ${ }^{3}$ Giancarlo Logroscino, ${ }^{5,6}$ \\ Orla Hardiman, ${ }^{\oplus 7}$ Elisabetta Pupillo, ${ }^{4}$ Jan H Veldink, ${ }^{\circledR 2}$ Roel Vermeulen, ${ }^{1,8}$ \\ Leonard $\mathrm{H}$ van den Berg, ${ }^{2}$ Euro-MOTOR consortium
}

\begin{abstract}
- Additional material is published online only. To view please visit the journal online (http://dx.doi.org/10.1136/ jnnp-2019-320986)
\end{abstract}

For numbered affiliations see end of article.

\section{Correspondence to}

Dr Susan Peters, Institute for Risk Assessment Sciences, Utrecht University, Utrecht 3584 CM, The Netherlands; s.peters@ uu.nl

RV and LHvdB contributed equally.

Received 11 April 2019 Revised 26 June 2019 Accepted 5 August 2019

\section{Check for updates}

(c) Author(s) (or their employer(s)) 2019. No commercial re-use. See rights and permissions. Published by BMJ.

To cite: Peters S, Visser AE, D'Ovidio F, et al. J Neurol Neurosurg Psychiatry Epub ahead of print: [please include Day Month Year]. doi:10.1136/jnnp-2019320986

\begin{abstract}
Background We investigated the association between cigarette smoking and risk of amyotrophic lateral sclerosis (ALS) in a pooled analysis of population-based case-control studies and explored the independent effects of intensity, duration and time-since-quitting. Methods ALS cases and controls, matched by age, sex and region, were recruited in the Netherlands, Italy and Ireland ( ${ }^{*}$ Euro-MOTOR project). Demographics and detailed lifetime smoking histories were collected through questionnaires. Effects of smoking status, intensity (cigarettes/day), duration (years), pack-years and time-since-quitting (years) on ALS risk were estimated using logistic regression models, adjusting for age, sex, alcohol, education and centre. We further investigated effect modification of the linear effects of pack-years by intensity, duration and time-since-quitting using excess $O R$ (eOR) models.
\end{abstract}

Results Analyses were performed on 1410 cases and 2616 controls. Pack-years were positively associated with ALS risk; OR=1.26 (95\% Cl: 1.03 to 1.54) for the highest quartile compared with never smokers. This association appeared to be predominantly driven by smoking duration $\left(p_{\text {trend }}=0.001\right)$ rather than intensity $\left(p_{\text {trend }}=0.86\right)$, although the trend for duration disappeared after adjustment for time-since-quitting. Time-since-quitting was inversely related to ALS ( $\left.p_{\text {trend }}<0.0001\right)$. The eOR decreased with time-since-quitting smoking, until about 10 years prior to disease onset. High intensity smoking with shorter duration appeared more deleterious than lower intensity for a longer duration.

Conclusions Our findings provide further support for the association between smoking and ALS. Pack-years alone may be insufficient to capture effects of different smoking patterns. Time-since-quitting appeared to be an important factor, suggesting that smoking may be an early disease trigger.

\section{INTRODUCTION}

Amyotrophic lateral sclerosis (ALS) is a fatal neurodegenerative disease. Patients typically die from respiratory failure, with a median survival of about 30 months following appearance of the first symptoms. ${ }^{1}$ Although the aetiology of ALS is still unclear, time and environmental exposures are thought to act on genetic pre-disposition until a turning point, when neurodegeneration starts. ${ }^{2}$

The role of exogenous factors in the risk of ALS is largely unknown; only cigarette smoking has been considered a probable risk factor. ${ }^{3}$ Several mechanisms have been proposed as to how cigarette smoking could increase the risk of ALS, including oxidant compounds and chemicals, such as lead and formaldehyde, which are present in smoke. ${ }^{4}$

We previously reported an increased risk of ALS among current smokers in a Dutch case-control study. ${ }^{5}$ Most detailed analyses of smoking parameters and the risk of ALS thus far, however, have been performed in a large European cohort ${ }^{6}$ and in five cohorts in the United States.

Overall, Gallo and colleagues found a nearly twofold increased risk of dying from ALS for those who were smokers at recruitment in comparison with never smokers. ${ }^{6}$ A clear relationship was observed between years spent smoking and ALS, with a stronger effect of smoking duration than intensity. ${ }^{6}$ Compared with continuing smoking, the number of years since smoking cessation was associated with a decreased risk. ${ }^{6}$

Wang and colleagues reported an increased ALS risk of about $40 \%$ for smokers versus never smokers. ${ }^{7}$ A clear linear trend for pack-years was found, with a positive association for both smoking intensity and duration, but these findings did not persist when never smokers were excluded. Among ever smokers, younger age at smoking initiation was associated with an increased risk of ALS. ${ }^{7}$

The estimation of independent effects of smoking metrics on disease risk is complex because duration, intensity and time-since-quitting are interrelated. The modifying effects of intensity and duration can, however, be assessed in a model that is conditioned on the linear effects of total exposure. ${ }^{8}$ This excess OR (eOR) model has recently been extended by including a direct assessment of time-since-quitting smoking and the model has been successfully applied in lung cancer ${ }^{9}$ and Parkinson's disease. ${ }^{10}$ Excess relative risk models have primarily been used in radiation epidemiology, but have thus far hardly been applied in rare disease case-control settings. ${ }^{11}$

Further exploration of the effects of different aspects of tobacco consumption on the risk of ALS 
may improve understanding of its aetiology. The aim of the current study was to investigate the risk of ALS associated with total pack-years of cigarette smoking in a pooled analysis of three population-based studies. The effect of smoking on ALS risk was further explored by investigating the independent contribution of duration, intensity and time-since cessation of smoking using eOR models.

\section{METHODS}

Population-based incident cases (18 years and above) with definite, probable or possible ALS, according to the revised *El Escorial Criteria, ${ }^{12}$ were recruited via population-based ALS registers in three European countries: the Netherlands (nationwide), Ireland (nationwide) and three regions in Italy (Apulia, Lombardy and Piedmont \& Valle d'Aosta) as part of the Euro-MOTOR project between 2010 and 2015. ${ }^{13}$ Controls were matched to the cases by sex, age ( \pm 5 years) and geographical location. In Ireland and Italy, controls were individually matched, and in the Netherlands they were frequency matched. Controls were identified via the local general practitioners. All participants gave written informed consent.

Lifetime smoking histories were obtained through paper questionnaires, using the same questions across centres. Face-to-face interviews were held in Ireland and Italy. In the Netherlands, questionnaires were self-administered and the participants were contacted by telephone to complete or clarify data when necessary. Smoking duration was the sum of years smoked, excluding the 3 years prior to the survey to eliminate any exposure that may have occurred after ALS diagnosis. Smoking intensity was the combined average number of cigarettes smoked per day during all smoking periods per subject. Subjects with an average intensity exceeding 60 cigarettes per day were excluded $(n=7)$, due to possible unreliable report of intensity. Cigarette packyears were calculated by dividing the intensity by 20 , multiplied by duration.

Unconditional logistic regression modelling was used to estimate the main effects for smoking. ORs and 95\% CIs were derived for smoking status (current, former, never smoker), smoking intensity (cigarettes per day), duration (in years), pack-years and time-since-quitting (in years). Categories were based on the quartiles of exposure distribution among controls. Models were adjusted for matching variables ${ }^{14}$ sex, age group ( $<50$ years, 50 to $<60,60$ to $<70,70$ to $<80$, and 80 and over) and centre (Ireland, Italy-Apulia, Italy-Lombardy, Italy-Piedmont \& Val d'Aosta, the Netherlands), as well as for alcohol drinking status (current, former, never drinker) and educational level (International Standard Classification of Education $0-4 ; 5-8^{15}$ ). The reference group for the categorical analyses consisted of never smokers. For time-since-quitting, current smokers served as the reference. $\mathrm{P}$ values for linear trends were obtained by including the respective continuous variable, among ever smokers only. In addition, we adjusted for the other smoking metrics, excluded former smokers from the analyses, stratified analyses by site of onset (ie, spinal or bulbar) and excluded cases with the highly penetrant C9orf72 repeat expansion ${ }^{16}$ to see if results changed. We also tested for possible heterogeneity in the observed associations by applying an interaction term between exposure metric and study centre. To explore possible residual confounding from educational level, we performed the same analyses on a post-hoc matched case-control set. ${ }^{17}$ Matching was done at an individual level for sex, age, centre and educational level, and conditional logistic regression modelling was applied, adjusted for alcohol drinking status.
The potential modification of the association between packyears and ALS by smoking intensity, duration and time-sincequitting was investigated in ever smokers using eOR modelling. These models allow for assessing the shape of the modification of the eOR per pack-year and can be (mathematically) described as follows:

$$
\begin{aligned}
& \mathrm{OR}(\mathrm{d})=1+\beta \mathrm{d} * \exp \left(\gamma_{1} \mathrm{~g}_{1} \text { (intensity) }\right) \\
& \mathrm{OR}(\mathrm{d})=1+\beta \mathrm{d} * \exp \left(\gamma_{2} \mathrm{~g}_{2} \text { (duration) }\right) \\
& \left.\mathrm{OR}(\mathrm{d})=1+\beta \mathrm{d} * \exp \left(\gamma_{3} \mathrm{~g}_{3} \text { (time }- \text { since }- \text { quitting }\right)\right) \\
& \mathrm{OR}(\mathrm{d})=1+\beta \mathrm{d} * \exp \left(\gamma_{1} \mathrm{~g}_{1} \text { (intensity) }+\gamma_{3} \mathrm{~g}_{3} \text { (time }- \text { since }- \text { quitting) }\right) \\
& \mathrm{OR}(\mathrm{d})=1+\beta \mathrm{d} * \exp \left(\gamma_{2} \mathrm{~g}_{2} \text { (duration) }+\gamma_{3} \mathrm{~g}_{3} \text { time }- \text { since }- \text { quitting) }\right)
\end{aligned}
$$

where $\beta$ is the eOR (defined as OR- ${ }^{*}$ ) for each pack-year $(d)$; $g_{1}, g_{2}$ and $g_{3}$ are restricted cubic spline functions with knots at the 20 th, 50 th and 80 th percentiles of the distribution of intensity, duration and time-since-quitting, respectively, and the $\gamma$ s are the spline coefficients. Alternative knot locations and number of knots were assessed, but we observed minimal effects on the model fit (based on the Akaike Information Criterion; AIC) and model prediction. All analyses were therefore performed with the a priori specified knots, similar to Vlaanderen et al (2014). ${ }^{9}$ We used PROC NLMIXED in SAS V.9.4 (SAS Institute, Cary, NC, USA) to fit the eOR models. ${ }^{18}$ Bootstrapping was used to estimate $95 \%$ CIs by calculating the 2.5 th and 97.5 th percentiles of the distribution for each parameter across 1000 bootstrap replications of the original data.

\section{RESULTS}

The study population comprised 1410 ALS cases (91\% of total cases in Euro-MOTOR) and 2616 controls (90\% of total) with complete smoking histories and information on potential confounders (table 1). Over $60 \%$ of subjects were ever smokers, with a median total smoking estimate of 13.6 pack-years among controls and 17.3 among cases. The medians for smoking intensity and duration were 12 cigarettes per day and 23 years for controls, and 14.2 cigarettes per day and 27 years for cases, respectively. The median time-since-quitting for former smokers was 25 years among controls and 22 among cases.

In the fully adjusted logistic regression models, smoking packyears showed a positive trend with ALS $\left(\mathrm{p}_{\text {trend }}=0.045\right)$ among ever smokers (table 2). The trend was stronger for smoking duration $\left(\mathrm{p}_{\text {trend }}=0.001\right)$ than for average smoking intensity $\left(\mathrm{p}_{\text {trend }}=0.86\right)$, when analysed separately. Mutual adjustment showed the same picture. The trend for smoking duration disappeared when the model was adjusted for time-since-quitting $\left(\mathrm{p}_{\text {trend }}=0.43\right)$ or when former smokers were excluded ( $\mathrm{p}_{\text {trend }}=0.97$, data not shown). A clear inverse relation with the risk of ALS $\left(p_{\text {trend }}<0.0001\right)$ was observed for time-since-quitting smoking, which remained after additional adjustment for pack-years $\left(\mathrm{p}_{\text {trend }}=0.0007\right)$. Analyses on the post-hoc matched case-control set resulted in virtually the same findings (table 2). Similar findings were observed when stratified by site of onset or when excluding cases with C9orf72 repeat expansions (online supplementary tables 1 and 2), and no significant heterogeneity between centres was observed (data not shown).

The eOR model that included spline functions for smoking duration and time-since-quitting provided the best fit to our data based on the AIC (data not shown). The highest risk per pack-year was observed for smoking durations of up to about 20 years (figure 1, left panel). Smoking for a shorter duration with high intensity appeared to be more deleterious than smoking for a longer duration with lower intensity. The eOR per pack-year 


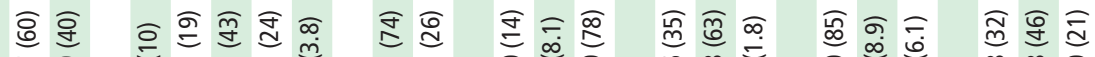

多染

䓂

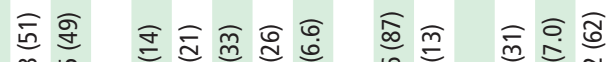

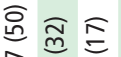

$\infty \quad$ cे

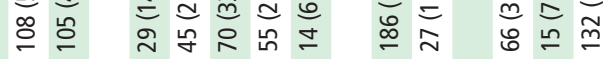

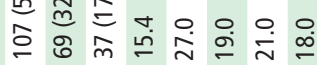

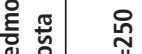

奐

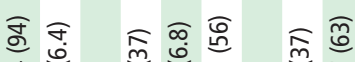

$\widehat{\widehat{c}}$

চ

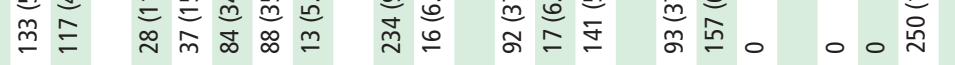

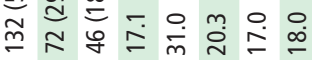

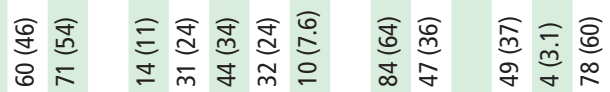

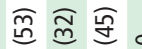

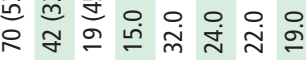

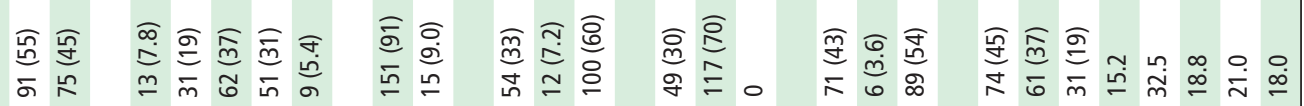
\&

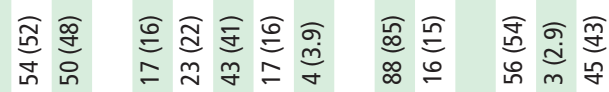

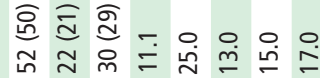

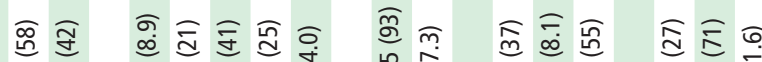

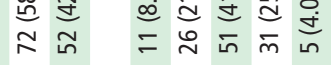

$\stackrel{n}{\circ}$

\&용

㭊 $\underset{\sim}{\sim}$

$\sigma \stackrel{0}{\stackrel{\circ}{a}}$

㠻空

일.

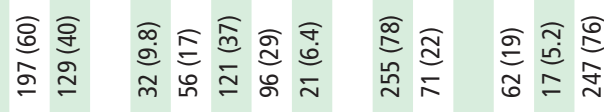

家 命

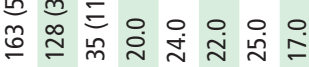

$+$

응

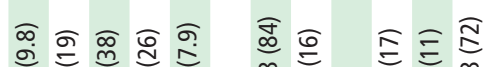

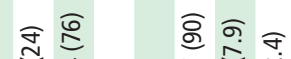

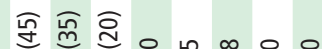

\%

$\stackrel{\infty}{=}$

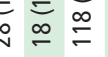

d

$\dot{m} \frac{a}{\mathfrak{d}}$

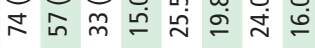

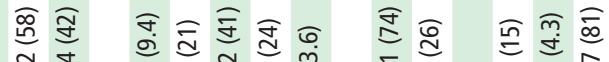

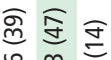

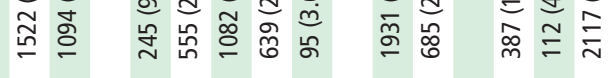

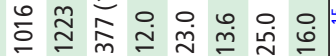

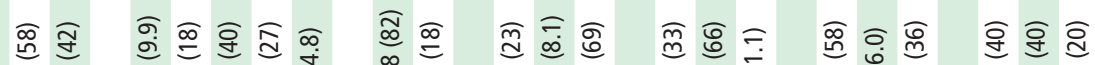

㢈
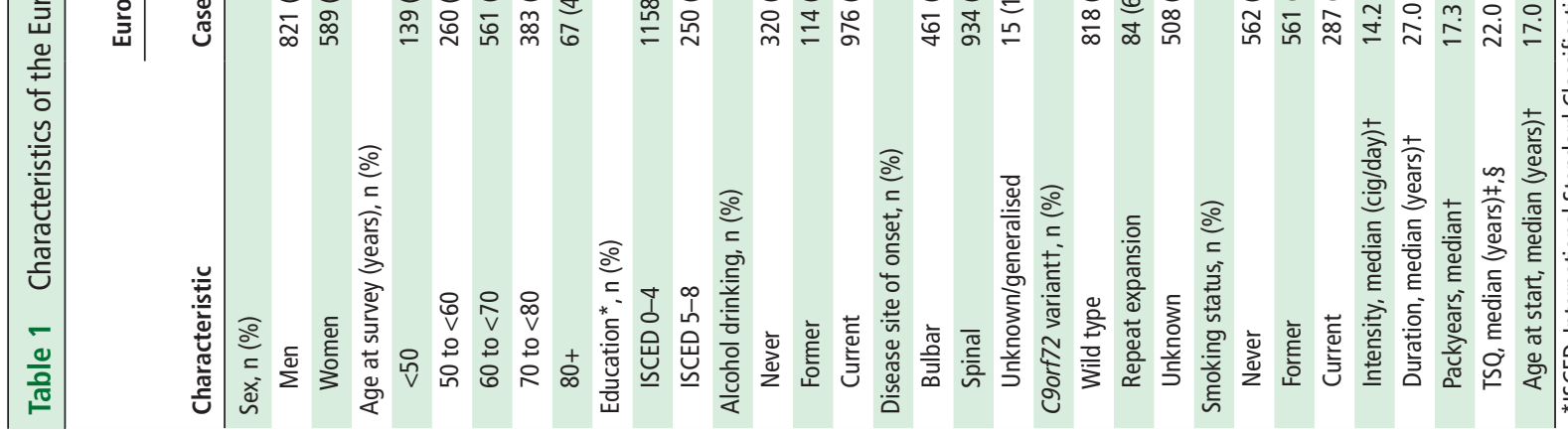


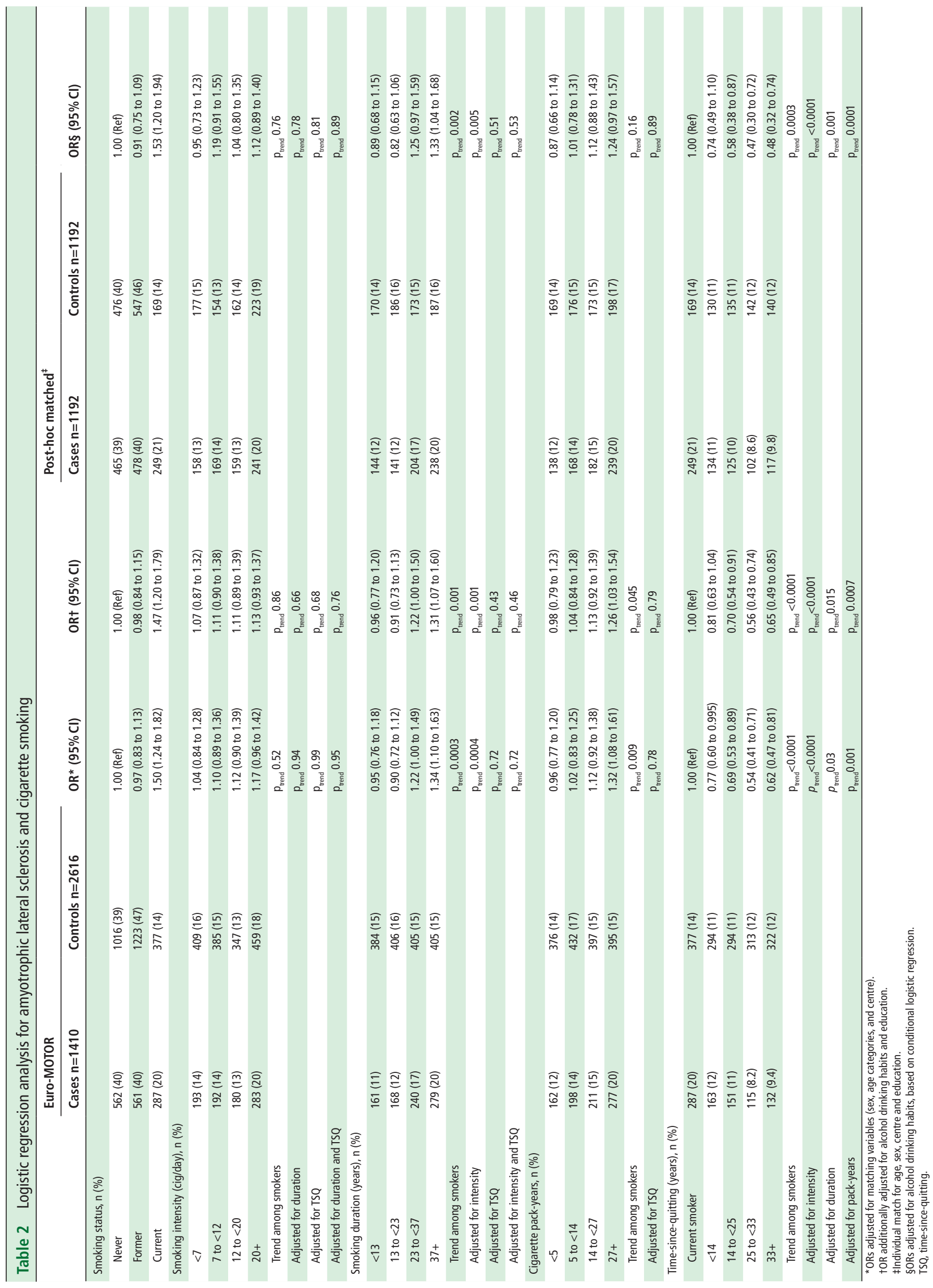



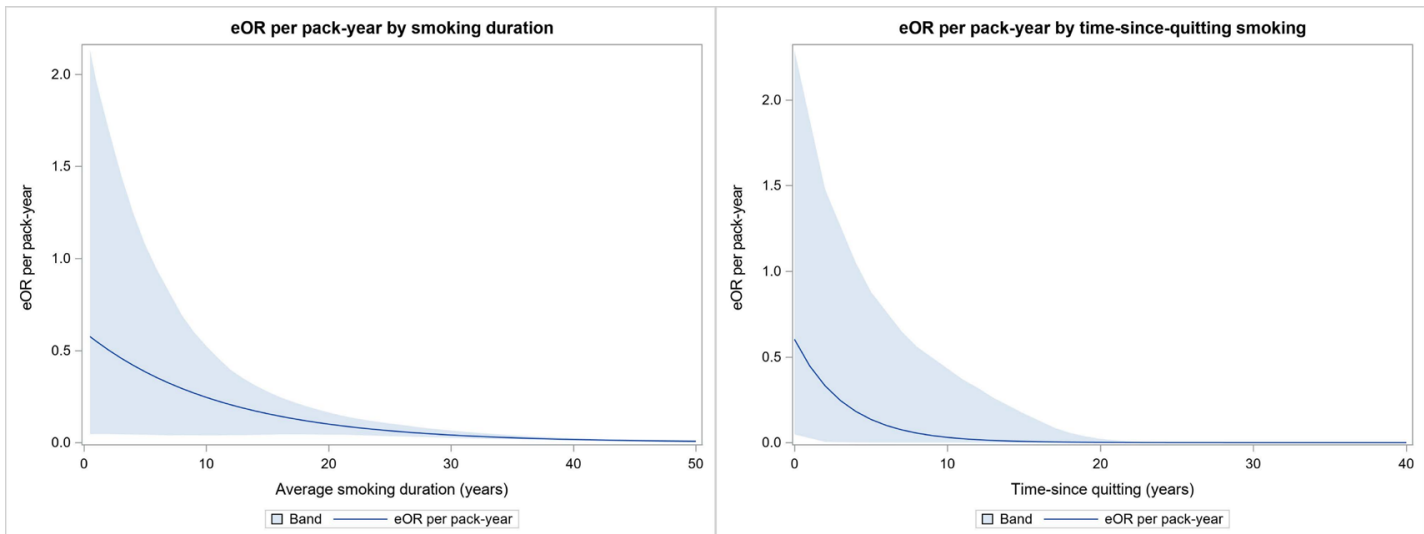

Figure 1 eOR for amyotrophic lateral sclerosis per pack-year smoking by duration (left panel) and time-since-quitting (right panel) in the Euro-MOTOR case-control study. Band $=95 \% \mathrm{Cl}$. eOR,excess OR.

steeply decreased with time-since-quitting smoking, until about 10 years prior to the disease onset (figure 1, right panel). Similar patterns were observed when stratified by site of onset of disease (data not shown).

\section{DISCUSSION}

A clear association between smoking and the risk of ALS was found in our pooled analysis of case-control studies from three European countries. Among ever smokers, we observed a continuous decrease in ALS risk with increasing time-sincequitting smoking. This effect was also evident in the eOR models that included the effects of cigarette pack-years. A similar pattern has previously been reported for the association between smoking and lung cancer. ${ }^{9}$ Furthermore, there was a suggestion that smoking many cigarettes per day for a shorter period was more deleterious than smoking fewer cigarettes per day for longer.

From the standard logistic regression models, intensity of smoking did not seem to affect the association between total smoking and risk of ALS. This association seemed to be mainly driven by duration of smoking, which is in line with previous findings in a large European cohort. ${ }^{6}$ However, when time-since-quitting was added to the model, or when former smokers were excluded, the trend for duration disappeared, indicating that time-since-quitting was the strongest predictor of risk. Smoking duration and time-since-quitting are strongly correlated (Pearson's $\mathrm{R}=-0.76$ ), which complicates disentangling their effects. ${ }^{19}$ The flexible eOR models are more suitable for this than standard logistic regression models, and results from our eOR models support the absence of an effect of smoking duration on the risk of ALS. Because time-sincequitting was not controlled for, the previous reported effect of smoking duration on $\mathrm{ALS}^{6}$ may well have been biassed.

Flexible eOR models allow for exploration of effect modification of the association of cumulative exposure (ie, pack-years) and risk of ALS by intensity, duration, and timesince-quitting smoking, while the effects of intensity and duration in a standard logistic regression is always distorted by the effect of cumulative exposure. As pack-years are the product of intensity and duration, a shorter smoking duration implies a higher number of cigarettes on average for the same total pack-years of smoking. We observed that the effect of one pack-year diminished with longer duration of smoking (and hence decreasing intensity) when time-since-quitting was also included, although CIs were wide. This observation suggests that intensity of smoking is influential on the association with ALS.

Previously, it has been suggested that six steps are needed to develop ALS, similar to cancer. ${ }^{20}$ These steps would include both genetic predisposition and environmental exposures, where the last exposure can be considered the disease trigger. The latency between exposure and disease onset is yet unknown. ${ }^{2}$ However, the large variability in disease progression $^{21}$ suggests that the pre-symptomatic phase may also be highly variable. The strong relationship with time-since-quitting and the result from the eOR model, suggest that smoking affects the risk of ALS most in the decade before onset. Hence, exposure to cigarette smoke could be an early trigger for disease.

Although the link between smoking and ALS is not as strong as for lung cancer, our findings show similar patterns emphasising the importance of taking into account when subjects quit smoking. Depending on the histological type, the excess risk of lung cancer after 11.5 to 14.4 years since quitting smoking is half of that associated with continued smoking. ${ }^{22}$ Based on our results, the risk of ALS seemed halved in about 5 years since quitting smoking.

Major strength of our case-control design is that we had population-based controls and we were able to include clinically confirmed ALS cases, instead of having to rely on the mention of motor neuron disease on death certificates. Moreover, the availability of detailed lifetime smoking histories for each subject enabled us to take into account smoking habits up to the start of the disease. The median time between diagnosis and survey completion was 15 months. We therefore chose to use 3 years before survey as a cut-off point for exposure to cigarette smoke, in order to eliminate recording of any exposure that may have occurred after the onset of ALS. In cohort studies, such information is typically only available up to baseline, which may ignore exposures and covariates many years prior to disease onset.

Our questionnaire allowed for reporting several periods of changing smoking habits and these changes were accounted for in the different metrics. In our models, however, we assigned the average number of cigarettes smoked per day, not taking into account when the changes in smoking intensity took place over time. The eOR model allowed us to disentangle the individual effects of both measures. When interpreting the results, however, one has to take into account the possibility that differential measurement error might affect the results. 
Duration of smoking may be more accurately estimated than intensity, although we have no indication that such misclassification of exposure would have been different between cases and controls in our study. Smoking history was one of various aspects that were included in the questionnaire, and subjects were not aware of the specific hypotheses tested.

We did not a priori exclude ALS cases with a C9orf72 repeat expansion, as it has been suggested that external factors (such as smoking) may still play a role on top of their genetic load. ${ }^{2}$ Limiting the analyses to cases with known wild type did not alter the overall picture. However, the risk of current smoking became somewhat more pronounced (OR 1.79, 95\% CI 1.41 to 2.27 ) as compared with the total group (OR 1.47, 95\% CI 1.20 to 1.79 ). Stratified analyses by site of onset revealed similar risks of smoking for both bulbar and spinal onset ALS.

Control subjects in our study population were overall more highly educated; $26 \%$ of controls were highly educated compared with $18 \%$ of ALS cases. We applied post-hoc matching for sex, age, centre and education to overcome this issue. The comparable findings indicate that the observed associations were unlikely to be affected by residual confounding from educational level.

We used population-based controls to limit selection bias. Among cases, however, there may have been some bias due to disease progression, if smoking is also associated with survival. Particularly fast progressors may be underrepresented, as patients are possibly less likely to complete a questionnaire when experiencing fast deterioration. If smoking affects the disease progression, assuming the effect is unfavourable, our findings are most likely an underestimation of the true effect.

A lower body-mass index (BMI) has been associated with an increased risk of ALS. ${ }^{23}$ Within Euro-MOTOR, information on weight was collected at recruitment and for every 10 year life stage. Since the causal association between body-mass and ALS is still unclear and BMI is highly affected by the disease process, BMI at recruitment was not informative in our analyses. Accurate information on weight prior to diagnosis was missing, so we were unable to adjust for possible confounding by premorbid BMI in the association between smoking and the risk of ALS.

In conclusion, our findings within the Euro-MOTOR case-control studies provide further support for the association between smoking and ALS. We showed a clear decreased risk of ALS with time-since-quitting smoking among smokers. Our findings further confirm that total smoking (expressed as pack-years) may not be sufficient to capture the effect of complex smoking patterns on disease risk. Identification of time-varying predictors of risk could provide insights into the dynamics of smoking-induced ALS. ${ }^{24}$ Understanding these dynamics may inform hypotheses on the disease mechanism and will enhance insight into the aetiology of ALS.

\section{Author affiliations}

'Institute for Risk Assessment Sciences, Utrecht University, Utrecht, The Netherlands

${ }^{2}$ Department of Neurology, University Medical Centre Utrecht, Utrecht, The Netherlands

3"Rita Levi Montalcini" Department of Neuroscience, University of Turin, Torino, Italy ${ }^{4}$ Department of Neuroscience, Istituto Mario Negri, Milan, Italy

${ }^{5}$ Unit of Neurodegenerative Diseases, Department of Clinical Research in Neurology, University of Bari "Aldo Moro", Pia Fondazione Cardinale G. Panico, Tricase, Lecce, Italy

${ }^{6}$ Department of Basic Medical Sciences, Neurosciences and Sense Organs, University of Bari "Aldo Moro", Bari, Italy

Academic Unit of Neurology, Trinity Biomedical Sciences Institute, Trinity College, Dublin, Ireland
${ }^{8}$ University Medical Centre Utrecht, Julius Centre for Public Health Sciences and Primary Care, Utrecht, The Netherlands

Collaborators Euro-MOTOR consortium: Anneke J van der Kooi; Joost Raaphorst; Andrea Calvo; Cristina Moglia; Federico Casale; Giuseppe Fuda; Antonio Canosa; Umberto Manera; Alessandro Bombaci; Maurizio Grassano; Rosario Vasta; Paolina Salamone; Giuseppe Marrali; Barbara Iazzolino; Letizia Mazzini; James Rooney; Mark Heverin; Alice Vajda; Giancarlo Comi; Nilo Riva; Christian Lunetta; Francesca Gerardi; Massimiliano Filosto; Maria Sofia Cotelli; Fabrizio Rinaldi; Luca Chiveri; Maria Cristina Guaita; Patrizia Perrone; Ceroni Mauro; Luca Diamanti; Carlo Ferrarese; Lucio Tremolizzo; Maria Luisa Delodovici; Giorgio Bono; Rosanna Tortelli; Chiara Zecca.

Contributors SP, AEV, FD, EB, AC, GL, OH, EP, JHV, RV and LvdB contributed to the study concept and design and participated in data collection and processing. SP performed the statistical analyses. SP, JV, LP and RV contributed to the analyses and interpretation of data. SP wrote the manuscript. AEV, FD, JV, LP, EB, AC, GL, OH, EP, $J H V, R V$ and $L v d B$ revised the manuscript for intellectual content. $E B, A C, G L, O H$, $J H V, R V$ and $L v d B$ provided study supervision. $E B, A C, G L, O H, J H V$ and $L v d B$ obtained funding.

Funding This work was supported by funding from the European Community's Health Seventh Framework Programme (FP7/2007-2013, grant number 259867); and the Netherlands ALS Foundation.

Competing interests EB reports grants from UCB-PHARMA, personal fees from MA-Provider, grants from American ALS Association, grants from EISAI, grants from Shire. AC reports personal fees from Biogen, grants from Italfarmaco, personal fees from Roche, personal fees from Cytokinetics, personal fees from Mitsubishi Tanabe. $\mathrm{OH}$ has received speaking honoraria from Novartis, Biogen Idec, Sanofi Aventis and Merck-Serono; and has been a member of advisory panels for Biogen Idec, Allergen, Ono Pharmaceuticals, Novartis, Cytokinetics and Sanofi Aventis. LvdB serves on scientific advisory boards for the Prinses Beatrix Spierfonds, Thierry Latran Foundation, Biogen, Cytokinetics, Orion and Sarepta. SP, AEV, FD, JV, LP, GL, EP, JHV and RV report no potential conflicts of interest.

Patient consent for publication Not required.

Provenance and peer review Not commissioned; externally peer reviewed. Data availability statement No data are available.

\section{REFERENCES}

1 Kiernan MC, Vucic S, Cheah BC, et al. Amyotrophic lateral sclerosis. The Lancet 2011:377:942-55.

2 Al-Chalabi A, Hardiman 0. The epidemiology of ALS: a conspiracy of genes, environment and time. Nat Rev Neurol 2013:9:617-28.

3 Armon C. Smoking may be considered an established risk factor for sporadic ALS. Neurology 2009;73:1693-8

4 Alonso A, Logroscino G, Hernán MA. Smoking and the risk of amyotrophic lateral sclerosis: a systematic review and meta-analysis. Journal of Neurology, Neurosurgery \& Psychiatry 2010;81:1249-52.

5 de Jong SW, Huisman MHB, Sutedja NA, et al. Smoking, alcohol consumption, and the risk of amyotrophic lateral sclerosis: a population-based study. Am J Epidemiol 2012;176:233-9.

6 Gallo V, Bueno-De-Mesquita HB, Vermeulen R, et al. Smoking and risk for amyotrophic lateral sclerosis: analysis of the EPIC cohort. Ann Neurol 2009;65:378-85.

7 Wang H, O'Reilly ÉJ, Weisskopf MG, et al. Smoking and risk of amyotrophic lateral sclerosis: a pooled analysis of 5 prospective cohorts. Arch Neurol 2011;68:13. doi:10.1001/archneurol.2010.367. [Epub ahead of print].

8 Lubin JH, Caporaso NE. Cigarette smoking and lung cancer: modeling total exposure and intensity. Cancer Epidemiol Biomarkers Prev 2006;15:517-23.

9 Vlaanderen J, Portengen L, Schüz J, et al. Effect modification of the association of cumulative exposure and cancer risk by intensity of exposure and time since exposure cessation: a flexible method applied to cigarette smoking and lung cancer in the synergy study. Am J Epidemiol 2014;179:290-8.

10 van der Mark M, Nijssen PCG, Vlaanderen J, et al. A case-control study of the protective effect of alcohol, coffee, and cigarette consumption on Parkinson disease risk: time-since-cessation modifies the effect of tobacco smoking. PLoS One 2014;9:e95297.

11 Lee W-C. Excess relative risk as an effect measure in case-control studies of rare diseases. PloS One 2014;10:e0121141.

12 Brooks BR, Miller RG, Swash M, et al. El Escorial revisited: revised criteria for the diagnosis of amyotrophic lateral sclerosis. Amyotroph Lateral Scler Other Motor Neuron Disord 2000;1:293-9.

13 D'Ovidio F, Rooney JPK, Visser AE, et al. Critical issues in ALS case-control studies: the case of the Euro-MOTOR study. Amyotroph Lateral Scler Frontotemporal Degener 2017:18:411-8

14 Pearce N. Analysis of matched case-control studies. BMJ 2016;352. 
15 UNESCO. International standard classification of education: ISCED 2011, 2011. Available: http://uis.unesco.org/sites/default/files/documents/internationalstandard-classification-of-education-isced-2011-en.pdf [Accessed 1 Nov 2017]

16 DeJesus-Hernandez M, Mackenzie IR, Boeve BF, et al. Expanded GGGGCC hexanucleotide repeat in noncoding region of C9orf72 causes chromosome 9p-linked FTD and ALS. Neuron 2011:72:245-56.

17 Visser AE, Rooney JPK, D'Ovidio F, et al. Multicentre, cross-cultural, populationbased, case-control study of physical activity as risk factor for amyotrophic lateral sclerosis. J Neurol Neurosurg Psychiatry 2018;89:797-803.

18 Richardson DB. A simple approach for fitting linear relative rate models in SAS. Am J Epidemiol 2008;168:1333-8.

19 Thomas DC. Invited commentary: is it time to retire the "pack-years" variable? Maybe not! Am J Epidemiol 2014;179:299-302.
20 Al-Chalabi A, Calvo A, Chio A, et al. Analysis of amyotrophic lateral sclerosis as a multistep process: a population-based modelling study. Lancet Neurol 2014;13:1108-13.

21 Swinnen B, RobberechtW. The phenotypic variability of amyotrophic lateral sclerosis. Nat Rev Neurol 2014:10:661-70

22 Fry JS, Lee PN, Forey BA, et al. How rapidly does the excess risk of lung cancer decline following quitting smoking? A quantitative review using the negative exponential model. Regul Toxicol Pharmacol 2013;67:13-26.

23 O'Reilly ÉJ, Wang M, Adami H-O, et al. Prediagnostic body size and risk of amyotrophic lateral sclerosis death in 10 studies. Amyotroph Lateral Scler Frontotemporal Degener 2018;19:406. doi:10.1080/21678421.2018.1452944. [Epub ahead of print].

24 Chadeau-Hyam M, Tubert-Bitter P, Guihenneuc-Jouyaux C, et al. Dynamics of the risk of smoking-induced lung cancer: a compartmental hidden Markov model for longitudinal analysis. Epidemiology 2014;25:28-34. 